\title{
Lectin Bead Array in a Single Tip Facilitates Fully
}

\section{Automatic Glycoprotein Profiling}

Hiroko Shimazaki $^{\dagger}$, Kozue Saito $^{\dagger}$, Atsushi Matsuda ${ }^{\dagger}, K_{\text {Kazumi Sawakami }}^{\ddagger}$, Minoru Kariya $a^{\ddagger}$, Osamu Segawa ${ }^{\ddagger}$, Yukiko Miyashita ${ }^{\ddagger}$, Tetsuya Ueda ${ }^{\ddagger}$, Michinori Koizuka ${ }^{\ddagger}$ Kazuhiro Nakamura ${ }^{\ddagger}$, Hiroyuki Kaji ${ }^{\dagger}$, Hideji Tajima and Atsushi Kuno*, ${ }^{\dagger}$

${ }^{\dagger}$ Glycoscience and Glycotechnology Research Group, Biotechnology Research Institute for Drug Discovery, National Institute of Advanced Industrial Science and Technology, Tsukuba, Ibaraki 305-8568, Japan.

¥Precision System Science, Kamihongou, Matsudo, Chiba 271-0064, Japan.

\section{* Corresponding author: Atsushi Kuno, PhD}

E-mail: atsu-kuno@aist.go.jp, Tel.: +81-29-861-3200, Fax: +81-29-861-3201

\section{Table of contents:}

- Materials

- Experimental procedures

- Supplementary tables (Table S1-S3)

- Supplementary figures (Fig. S1-S10)

- References p. $\mathrm{S} 2$

pp. S2 - S4

pp. S5 - S7

pp. $\mathrm{S} 8-\mathrm{S} 18$

p. S19 


\section{Materials}

Lectins were purchased from J-Chemicals (Tokyo, Japan), Vector Laboratories (Burlingame, CA, USA), or Fujifilm Wako Pure Chemical Co (Osaka, Japan). Sialidase A and peptide- $N$-glycosidase F (PNGaseF) were purchased from ProZyme (Hayward, CA, USA) and Takara Bio. (Shiga, Japan), respectively. Mouse anti-human erythropoietin was purchased from Bio-Rad Laboratories (Hercules, CA, USA). Human serum from a healthy volunteer was purchased from Kohjin Bio (Saitama, Japan).

\section{Experimental procedures}

\section{Mass spectrometry (MS) analysis of released $\mathrm{N}$-Glycans}

All $N$-glycans were released from the tryptic digest of reduced and alkylated protein sample with peptide- $N$-glycosidase F. Released glycans were applied to an OASIS HLB cartridge (Waters, Milford, MA, USA) and the pass fraction was recovered. The glycans were collected with a PGC cartridge (HyperSep Hypercarb, Thermo Scientific, USA) and permethylated with methyl iodide. After cleaning with a Sep Pak C18 cartridge (Waters), the glycans were mixed with a matrix solution (2,5-dihydroxybenzoic acid (DHB, Fujifilm Wako Pure Chemical Co) on a stainlesssteel sample plate for MALDI MS. The mass spectrum was obtained using an Ultraflex TOF/TOF (Bruker, Billerica, MA, USA) system in reflectron positive mode. The spectrum was analyzed using flexAnalysis software (version 2.0, Bruker). Observed signals were assigned by comparison with the observed mass value and calculated masses of the permethylated glycans, where all glycan masses were calculated as sodium adducts.

\section{Fabrication of the GlycoBIST platform}

Spacer beads were made by immobilizing BSA on silicon carbide beads (Precision System Science, Matsudo, Japan): 2000-4000 beads were simultaneously washed twice with wash buffer, then incubated with $10 \mathrm{mg} / \mathrm{mL}$ BSA in PBS for $16-24 \mathrm{~h}$ at $4{ }^{\circ} \mathrm{C}$ with gentle shaking. The resultant beads were washed three times with wash buffer and incubated in StabilGuard Immunoassay Stabilizer (BSA-Free) (SurModics, Eden Prairie, MN, USA) for $1 \mathrm{~h}$ at room temperature. The coated beads with BSA were put onto a Micro Bio-Spin chromatography column and centrifuged to remove solutions as for the reaction beads. The remaining steps including drying and storing were also the same as for the reaction beads. To construct a GlycoBIST, up to 10 reaction beads were arranged into a GlycoBIST capillary inserting spacer beads at defined intervals (Fig. 1A). Both the 
bottom and top ends of the capillary were pinched weakly using a dedicated pincer not to lose beads. The completed GlycoBISTs were kept in a sealed bag with a desiccant at $4{ }^{\circ} \mathrm{C}$ until just before use.

\section{Multilectin assay by GlycoBIST}

To visualize the glycoprotein-lectin interaction, an analyte glycoprotein was labeled with Cy3-succinimidyl ester in advance. After the binding reaction of Cy3-labeled glycoprotein performed with the Purelumn system, one end of the capillary tip was cut, and beads reacted with $\mathrm{Cy} 3$-glycoprotein were taken out from the tip, and the distribution of the Cy3-glycoprotein on the beads was confirmed with a fluorescence microscope (BZX710; Keyence, Osaka, Japan).

To measure endogenous glycoproteins, an 8-plex advanced equipment named LuBEA GT8 (Precision System Science), which is an automation system performing the processes from binding reaction to chemiluminescent detection, was used.

\section{Antibody-overlay lectin microarray}

An analyte glycoprotein, which was commercially available or immunoprecipitated from serum or cell lysate, was diluted with PBS containing 1\% Triton X-100 (PBSTx) and applied to a lectin array slide LecChip (GlycoTechnica, Yokohama, Japan), then incubated for $16 \mathrm{~h}$ at $20^{\circ} \mathrm{C}$. After the incubation, human serum polyclonal $\operatorname{IgG}(20 \mu \mathrm{g})$ was added and further incubated for $30 \mathrm{~min}$ at $20{ }^{\circ} \mathrm{C}$ (to reduce background noise). After washing three times with PBSTx, 50-150 ng of biotinylated antibody against the targeted protein in PBSTx was added and incubated for $60 \mathrm{~min}$ at $20{ }^{\circ} \mathrm{C}$. After the slide was washed three times with PBSTx, 100-300 ng of a Cy3-labeled streptavidin (GE Healthcare, Little Chalfont, UK) in PBSTx was added to the slide and then incubated for 30 min at $20^{\circ} \mathrm{C}$. After washing three times with PBSTx, the slide was scanned with an evanescent-field fluorescence scanner (GlycoStation Reader 1200; GlycoTechnica). All data were analyzed with GlycoStation ToolsPro, version 1.5 (GlycoTechnica) and the net signal intensity of each spot was calculated by subtracting the background value from the average signal intensity of three spots.

\section{Lectin-antibody sandwich ELISA}

In brief, $250 \mathrm{ng}$ of biotin-conjugated lectin was coated on a streptavidin-precoated microtiter plate (Thermo Fisher Scientific, Fremont, CA, USA) and kept overnight at room temperature. After washing twice with PBS containing $0.1 \%$ Tween 20 (PBST), the analyte protein solution was applied to the plate and incubated for $60 \mathrm{~min}$ at $37^{\circ} \mathrm{C}$. After 
incubation with the analyte protein, human serum polyclonal IgG $(20 \mu \mathrm{g})$ was added and then incubated for $30 \mathrm{~min}$ at $37^{\circ} \mathrm{C}$ to reduce background noise. After washing five times with PBST, $10 \mathrm{ng}$ of horseradish peroxidase (HRP)-conjugated anti-erythropoietin antibody (in $50 \mu \mathrm{L}$ of Can Get Signal Solution 1; Toyobo, Osaka, Japan) was added and incubated with the mixture for $30 \mathrm{~min}$ at $37^{\circ} \mathrm{C}$. After washing five times with PBST, 100 $\mu \mathrm{L}$ of the substrate solution 3,3',5,5'-tetramethylbenzidine (Thermo Fisher Scientific) was added to each well and reacted with the HRP for $15 \mathrm{~min}$ at $37^{\circ} \mathrm{C}$. The oxidation reaction was stopped by adding $100 \mu \mathrm{L}$ of $1 \mathrm{M}$ sulfuric acid, and OD was measured at $450 \mathrm{~nm}$.

\section{Preparation of endogenous glycoproteins}

AGP was enriched from serum using streptavidin magnetic beads (Dynabeads, Life Technologies, Carlsbad, CA, USA). Anti-AGP monoclonal antibody (Sigma-Aldrich, St. Louis, MO, USA) was biotinylated using Biotin Labeling Kit-NH2 (Dojindo Laboratories, Kumamoto, Japan) and incubated with $0.5 \mu \mathrm{L}$ of heat-inactivated serum from a healthy volunteer for $30 \mathrm{~min}$ at $4{ }^{\circ} \mathrm{C}$. Antibody-antigen complex was further incubated with streptavidin-coated magnetic beads in TBSTx for $30 \mathrm{~min}$ at $4{ }^{\circ} \mathrm{C}$. After washing three times with TBSTx, $10 \mu \mathrm{L}$ of TBS containing $0.2 \%$ SDS was added to the beads and incubated for $5 \mathrm{~min}$ at $60{ }^{\circ} \mathrm{C}$. Supernatants were collected as AGP immunoprecipitated samples. Membrane-tethered MUC1 was prepared from a lysate of the pancreatic cancer cell line Capan-1. We disrupted $1 \times 10^{6}$ cells by gentle sonication in $1 \%$ NP40, then centrifuged the suspension at $20,000 \times g$ for $30 \mathrm{~min}$ at $4{ }^{\circ} \mathrm{C}$. The resultant supernatants were collected as a protein extract sample of Capan-1. Biotinylated anti-sialylated MUC1 monoclonal antibody, MY.1E12 (500 ng), was conjugated with $200 \mu \mathrm{g}$ of streptavidincoated magnetic beads and incubated with one-fifth of the protein extraction sample of Capan-1 lysate for $16 \mathrm{~h}$ at $4{ }^{\circ} \mathrm{C}$. After washing three times with PBSTx, $10 \mu \mathrm{L}$ of PBS containing $0.2 \%$ SDS was added to the beads and bound materials were eluted by heat denaturation for $5 \mathrm{~min}$ at $95{ }^{\circ} \mathrm{C}$. Supernatants were further incubated with $400 \mu \mathrm{g}$ of streptavidin-coated magnetic beads for $30 \mathrm{~min}$ at $4{ }^{\circ} \mathrm{C}$ to remove contaminant biotinylated MY.1E12. 


\section{Supplementary Tables}

Table S1. List of conditions for immobilization of lectin to reaction beads.

\begin{tabular}{|l|c|c|c|c|}
\hline & \multicolumn{2}{|c|}{ for EPO } & \multicolumn{2}{c|}{ for AGP, MUC1 } \\
\hline & $\begin{array}{c}\text { conc. } \\
\text { (mg/mL) }\end{array}$ & $\mathrm{pH}$ & $\begin{array}{c}\text { conc. } \\
(\mathrm{mg} / \mathrm{mL})\end{array}$ & $\mathrm{pH}$ \\
\hline DSA & 0.05 & 7.4 & 0.1 & 7.4 \\
\hline PHA-E & 0.05 & 7.4 & 0.1 & 7.4 \\
\hline MAL & 0.05 & 4.0 & 0.1 & 4.0 \\
\hline ECA & 0.05 & 7.4 & 0.1 & 4.0 \\
\hline PHA-L & 0.05 & 7.4 & - & - \\
\hline WFA & 0.05 & 7.4 & 0.1 & 7.4 \\
\hline BPL & 0.05 & 7.4 & - & - \\
\hline ABA & 0.05 & 4.0 & 0.5 & 4.0 \\
\hline MAH & 0.05 & 7.4 & - & - \\
\hline SSA & 0.05 & 7.4 & 0.5 & 7.4 \\
\hline LEL & - & - & 0.1 & 7.4 \\
\hline ConA & - & - & 0.1 & 7.4 \\
\hline RCA120 & - & - & 0.1 & 7.4 \\
\hline WGA & - & - & 0.5 & 4.0 \\
\hline Jacalin & - & - & 0.5 & 7.4 \\
\hline MPA & - & - & 0.5 & 4.0 \\
\hline AAL & - & - & 0.1 & 7.4 \\
\hline
\end{tabular}


Table S2. Mass assignments of permethylated $N$-glycans released from erythropoietin samples.

\begin{tabular}{|c|c|c|c|c|c|c|c|c|c|c|c|}
\hline \multicolumn{2}{|c|}{ D-EPO Sialidase(-) } & \multirow[b]{2}{*}{$\begin{array}{l}\Delta \text { mass } \\
\text { (Dalton) }\end{array}$} & \multirow[b]{2}{*}{$\begin{array}{c}\text { Glycan } \\
\text { composition } * *\end{array}$} & \multirow[b]{2}{*}{ Intensity } & \multirow[b]{2}{*}{$\begin{array}{c}\text { Relative } \\
\text { intensity }(\%) \\
\end{array}$} & \multicolumn{2}{|c|}{ EPO- $\beta$ Sialidase $(-)$} & \multirow[b]{2}{*}{$\begin{array}{l}\text { mass } \\
\text { (Dalton) }\end{array}$} & \multirow[b]{2}{*}{$\begin{array}{c}\text { Glycan } \\
\text { composition } * *\end{array}$} & \multirow[b]{2}{*}{ Intensity } & \multirow[b]{2}{*}{$\begin{array}{c}\text { Relative } \\
\text { intensity (\%) }\end{array}$} \\
\hline $\begin{array}{c}\text { Observed } \\
\mathrm{m} / \mathrm{z}\end{array}$ & $\begin{array}{c}\text { Calculated } \\
\mathrm{m} / \mathrm{z} *\end{array}$ & & & & & $\begin{array}{c}\text { Observed } \\
\mathrm{m} / \mathrm{z}\end{array}$ & $\begin{array}{c}\text { Calculated } \\
\mathrm{m} / \mathrm{z} *\end{array}$ & & & & \\
\hline & & & & & & 2605.36 & 2605.30 & 0.07 & $221-1$ & 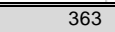 & 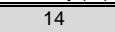 \\
\hline 2966.81 & 2966.47 & 0.34 & $221-2$ & 679 & 15 & 2966.52 & 2966.47 & 0.05 & $221-2$ & 809 & 32 \\
\hline 3416.14 & 3415.70 & 0.44 & $331-2$ & 627 & 14 & 3415.83 & 3415.70 & 0.13 & $331-2$ & 624 & 24 \\
\hline 3777.43 & 3776.87 & 0.56 & $331-3$ & 1,541 & 34 & 3777.13 & 3776.87 & 0.26 & $331-3$ & 1,422 & 56 \\
\hline 3865.51 & 3864.92 & 0.59 & $441-2$ & 553 & 12 & 3865.20 & 3864.92 & 0.28 & $441-2$ & 577 & 23 \\
\hline \multirow[t]{3}{*}{4226.87} & 4226.10 & 0.77 & $441-3$ & 1,425 & 32 & 4226.57 & 4226.10 & 0.48 & $441-3$ & 1,888 & 74 \\
\hline & & & & & & 4314.62 & 4314.15 & 0.47 & $551-2$ & 341 & 13 \\
\hline & & & & & & 4400.68 & 4400.19 & 0.49 & $442-3$ & 297 & 12 \\
\hline \multirow[t]{3}{*}{4588.29} & 4587.27 & 1.02 & $441-4$ & 4,484 & 100 & 4587.99 & 4587.27 & 0.71 & $441-4$ & 2,551 & 100 \\
\hline & & & & & & 4676.09 & 4675.32 & 0.76 & $551-3$ & 677 & 27 \\
\hline & & & & & & 4762.17 & 4761.36 & 0.81 & $442-4$ & 269 & 11 \\
\hline \multirow[t]{4}{*}{5037.76} & 5036.50 & 1.26 & $551-4$ & 391 & 9 & 5037.58 & 5036.50 & 1.08 & $551-4$ & 790 & 31 \\
\hline & & & & & & 5125.76 & 5124.55 & 1.21 & $661-3$ & 301 & 12 \\
\hline & & & & & & 5487.20 & 5485.72 & 1.47 & $661-4$ & 316 & 12 \\
\hline & & & & & & & & & & & \\
\hline \multicolumn{2}{|c|}{ D-EPO Sialidase (+) } & & & & & \multicolumn{2}{|c|}{ EPO- $\beta$ Sialidase (+) } & & & & \\
\hline \multirow[t]{2}{*}{$\begin{array}{c}\text { Observed } \\
\mathrm{m} / \mathrm{z}\end{array}$} & Calculated & $\begin{array}{l}\Delta \text { mass } \\
\text { (Dalton) }\end{array}$ & $\begin{array}{c}\text { Glycan } \\
\text { composition } * *\end{array}$ & Intensity & $\begin{array}{c}\text { Relative } \\
\text { intensity (\%) }\end{array}$ & Observed & Calculated & $\begin{array}{l}\Delta \text { mass } \\
\text { (Dalton) }\end{array}$ & $\begin{array}{c}\text { Glycan } \\
\text { composition } * *\end{array}$ & Intensity & $\begin{array}{c}\text { Relative } \\
\text { intensity (\%) }\end{array}$ \\
\hline & & & & & & 2244.16 & 2244.12 & 0.03 & 221 & 812 & 5 \\
\hline \multirow[t]{2}{*}{2693.47} & 2693.35 & 0.12 & 331 & 2,170 & 31 & 2693.36 & 2693.35 & 0.01 & 331 & 3,095 & 19 \\
\hline & & & & & & 2938.45 & 2938.48 & -0.03 & 341 & 241 & 1 \\
\hline \multirow[t]{2}{*}{3142.80} & 3142.58 & 0.23 & 441 & 7,063 & 100 & 3142.64 & 3142.58 & 0.06 & 441 & 16,421 & 100 \\
\hline & & & & & & 3316.78 & 3316.67 & 0.11 & 442 & 326 & 2 \\
\hline \multirow[t]{4}{*}{3592.07} & 3591.80 & 0.27 & 551 & 1,246 & 18 & 3592.01 & 3591.80 & 0.21 & 551 & 4,938 & 30 \\
\hline & & & & & & 3766.26 & 3765.89 & 0.37 & 552 & 210 & 1 \\
\hline & & & & & & 4041.43 & 4041.03 & 0.40 & 661 & 1,036 & 6 \\
\hline & & & & & & 4490.86 & 4490.26 & 0.61 & 771 & 209 & 1 \\
\hline & & & & & & & & & & & \\
\hline${ }^{*}$ Calculated & Is sodium ad & duct of the & assigned glyc & & & & & & & & \\
\hline
\end{tabular}


Table S3. Reproducibility test. A: simultaneous reproducibility test in five different tips.

B: Estimation of run-to-run variation of five runs.

A

\begin{tabular}{|c|c|c|c|}
\hline & \multicolumn{3}{|c|}{ EPO- $\beta$, Sialidase A (-) } \\
\hline & AVERAGE & SD & CV(\%) \\
\hline DSA & 627.17 & 6.80 & 1.08 \\
\hline PHA-E & 154.84 & 4.36 & 2.81 \\
\hline MAL & 86.39 & 2.38 & 2.76 \\
\hline ECA & 10.29 & 0.62 & 6.00 \\
\hline PHA-L & 47.90 & 3.08 & 6.43 \\
\hline WFA & ND & ND & ND \\
\hline BPL & ND & ND & ND \\
\hline ABA & 33.03 & 1.45 & 4.39 \\
\hline MAH & 21.43 & 1.40 & 6.54 \\
\hline SSA & 12.93 & 1.02 & 7.92 \\
\hline
\end{tabular}

\begin{tabular}{|c|c|c|c|}
\hline & \multicolumn{3}{|c|}{ EPO- $\beta$, Sialidase A (+) } \\
\hline & AVERAGE & SD & CV $(\%)$ \\
\hline DSA & 456.65 & 11.24 & 2.46 \\
\hline PHA-E & 94.03 & 5.61 & 5.97 \\
\hline MAL & ND & ND & ND \\
\hline ECA & 106.90 & 3.13 & 2.93 \\
\hline PHA-L & 99.82 & 1.96 & 1.97 \\
\hline WFA & 92.82 & 3.26 & 3.51 \\
\hline BPL & 56.30 & 3.32 & 5.89 \\
\hline ABA & 53.33 & 3.95 & 7.40 \\
\hline MAH & ND & ND & ND \\
\hline SSA & 22.47 & 0.46 & 2.06 \\
\hline
\end{tabular}

B

\begin{tabular}{|c|c|c|c|c|c|c|c|c|}
\hline & \multicolumn{3}{|c|}{ EPO- $\beta$, Sialidase A (-) } & & \multicolumn{3}{c|}{ EPO- $\beta$, Sialidase A (+) } \\
\cline { 5 - 8 } & AVERAGE & SD & CV(\%) & & AVERAGE & SD & CV(\%) \\
\hline DSA & 575.68 & 36.85 & 6.40 & DSA & 440.99 & 16.19 & 3.67 \\
\hline PHA-E & 191.84 & 24.03 & 12.52 & PHA-E & 111.36 & 13.06 & 11.72 \\
\hline MAL & 90.88 & 16.37 & 18.01 & MAL & ND & ND & ND \\
\hline ECA & ND & ND & ND & ECA & 113.95 & 16.05 & 14.08 \\
\hline PHA-L & 53.03 & 4.47 & 8.44 & PHA-L & 100.04 & 10.09 & 10.08 \\
\hline WFA & ND & ND & ND & WFA & 85.62 & 4.19 & 4.89 \\
\hline BPL & ND & ND & ND & BPL & 52.30 & 4.21 & 8.04 \\
\hline ABA & 38.11 & 5.11 & 13.42 & ABA & 56.97 & 5.84 & 10.24 \\
\hline MAH & 23.49 & 1.64 & 6.97 & MAH & ND & ND & ND \\
\hline SSA & 12.28 & 0.77 & 6.30 & SSA & 22.30 & 0.68 & 3.04 \\
\hline
\end{tabular}




\section{Supplementary figures}

A

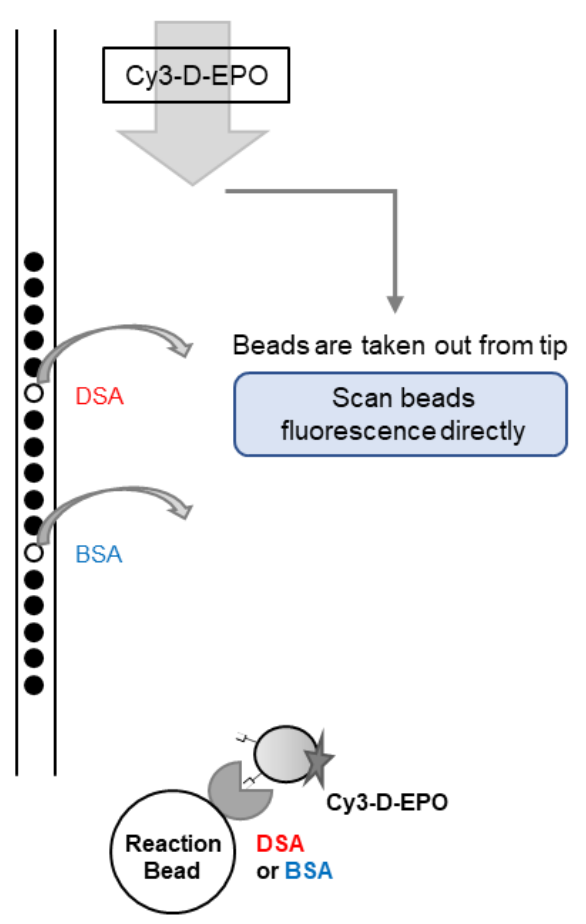

B

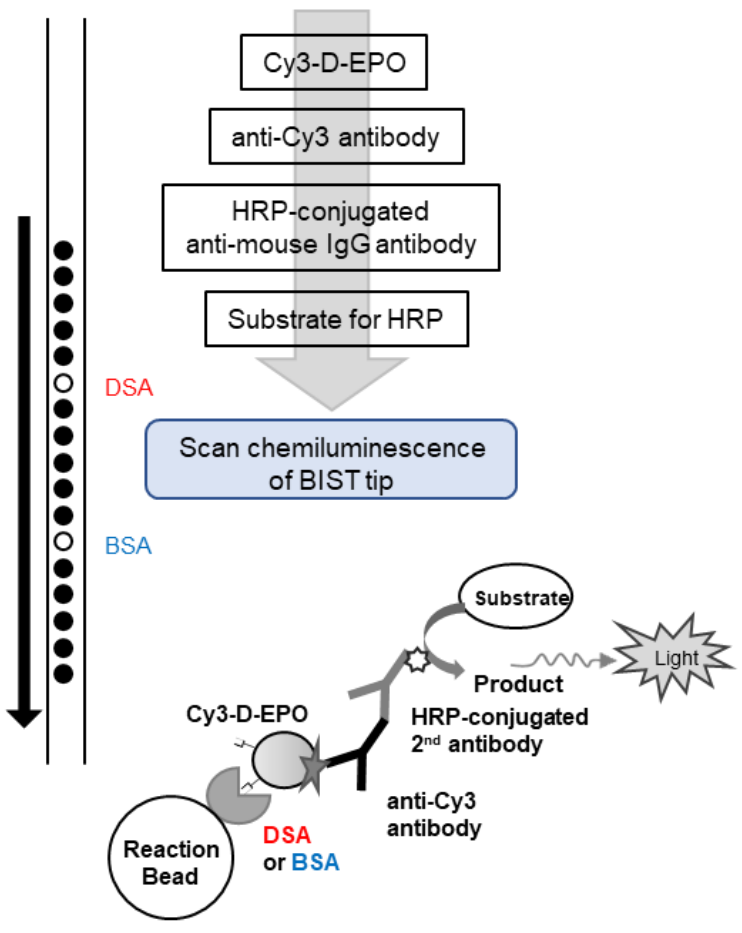

Figure S1. Schematic overview of two sets of experiments to verify the glycoproteinlectin interaction. Six same sets of GlycoBIST were prepared and half of them were used for interaction only with Cy3-labeled EPO (A), and the other half were used for sequential interaction with Cy3-EPO, anti-Cy3 antibody, the second antibody labeled with HRP and chemiluminescent substrate solution (B). In experiment A, binding was confirmed more clearly by direct observation of fluorescence distribution on reaction beads taken from the tip. In experiment B, the amount of binding of Cy3-EPO was obtained as a chemiluminescent signal scanned from the top to the bottom of the tip. 


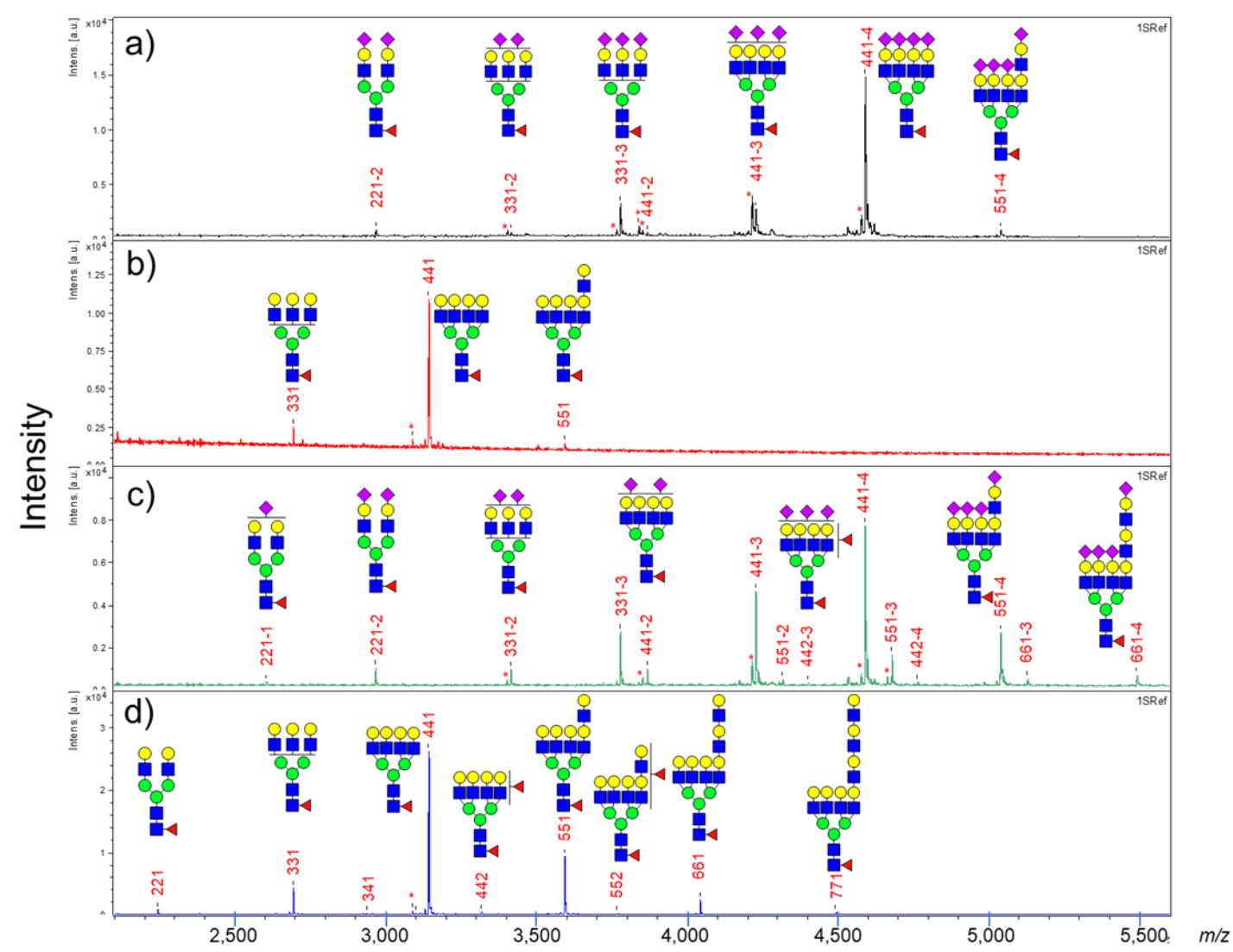

Figure S2. Mass assignments of permethylated $N$-glycans released from erythropoietin samples. (a) D-EPO, (b) D-EPO treated with sialidase A, (c) EPO- $\beta$, (d) EPO- $\beta$ treated with sialidase A. The number on each signal (XYZ-S) is the assigned glycan composition of Hex, HexNAc, dHex, and NeuAc, respectively, on the trimannosyl core $($ Hex3HexNAc2 $=000)$. The signal assignment was based on the mass value of permethylated glycans calculated as sodium adducts. The plausible glycan structure is shown over each signal. Yellow circle: galactose, blue square: $N$-acetylglucosamine, green circle: mannose, red triangle: fucose, purple diamond: $N$-acetylneuraminic acid. *presumed to be signals in which methylation is incomplete. 


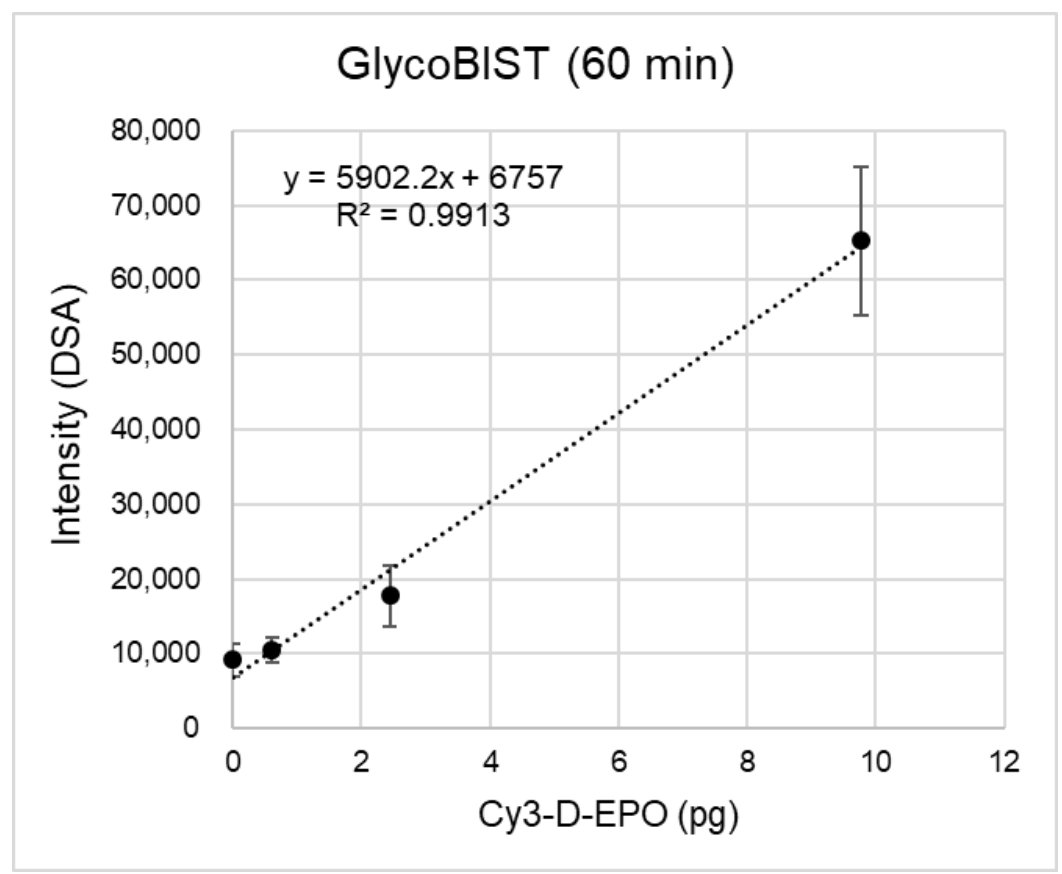

Figure S3. Dose dependency of the chemiluminescent signal of GlycoBIST interacted with Cy3-D-EPO for $60 \mathrm{~min}$. 


\begin{tabular}{|c|c|c|c|c|c|}
\hline $\begin{array}{l}\text { Cy3- } \\
\text { D-EPO } \\
\text { (ng) }\end{array}$ & Gain 105 & Gain95 & Gain 85 & Gain75 & Gain 65 \\
\hline 20 & 61,000 & 63,900 & 64,500 & 53,400 & 36,600 \\
\hline 5 & 62,600 & 64,600 & 63,300 & 48,000 & 32,400 \\
\hline 1.25 & 63,000 & 62,700 & 47,100 & 31,300 & 20,600 \\
\hline 0.313 & 40,300 & 25,300 & 16,400 & 10,100 & 6,500 \\
\hline 0.078 & 9,700 & 4,800 & 3,000 & 2,000 & 1,300 \\
\hline 0.020 & 2,200 & 1,000 & 500 & 300 & 400 \\
\hline 0 & 200 & 0 & 0 & 0 & 0 \\
\hline
\end{tabular}

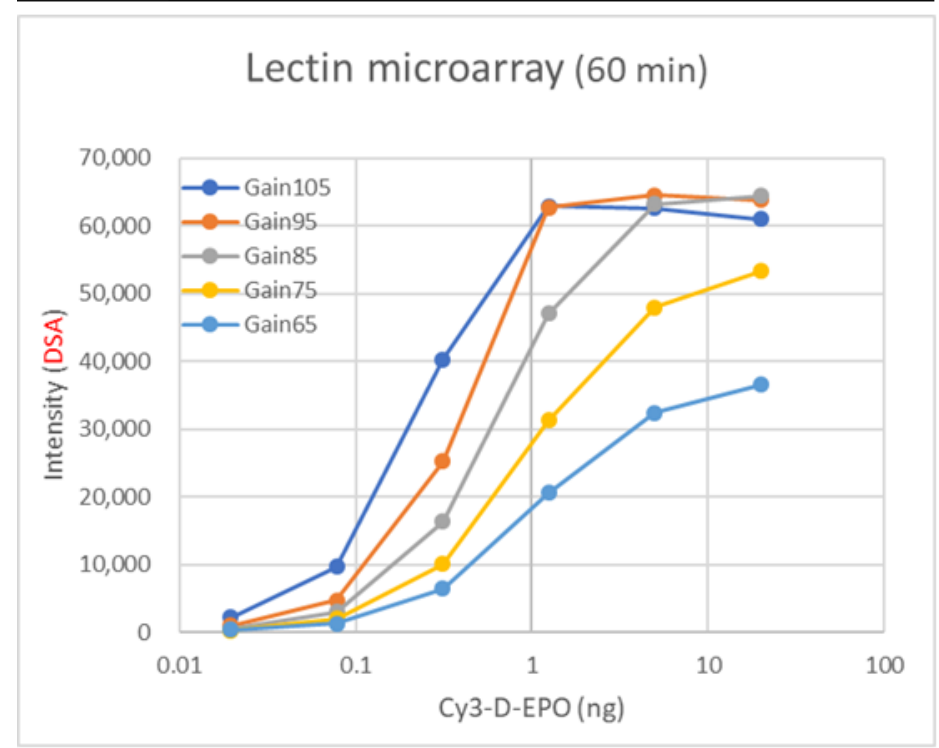

Figure S4. Dose dependency of the fluorescent signal of the DSA spot on the lectin microarray interacted with Cy3-D-EPO for $60 \mathrm{~min}$. The scanning data under appropriate gain conditions (Gain65) were selected for Fig.2C, which provided the net intensities of all positive spots $<40,000$. Gray indicates out of detection range. 
A

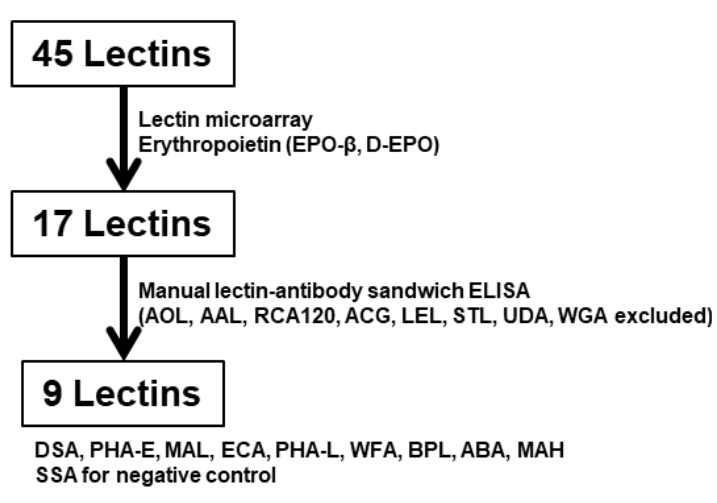

C

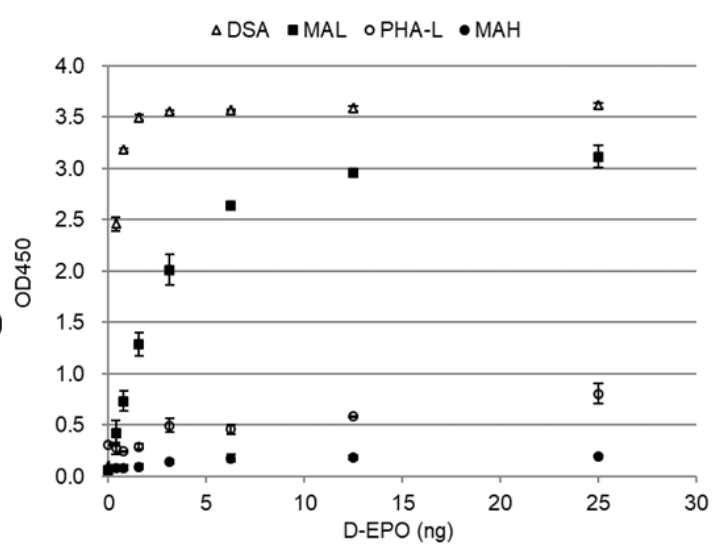

B
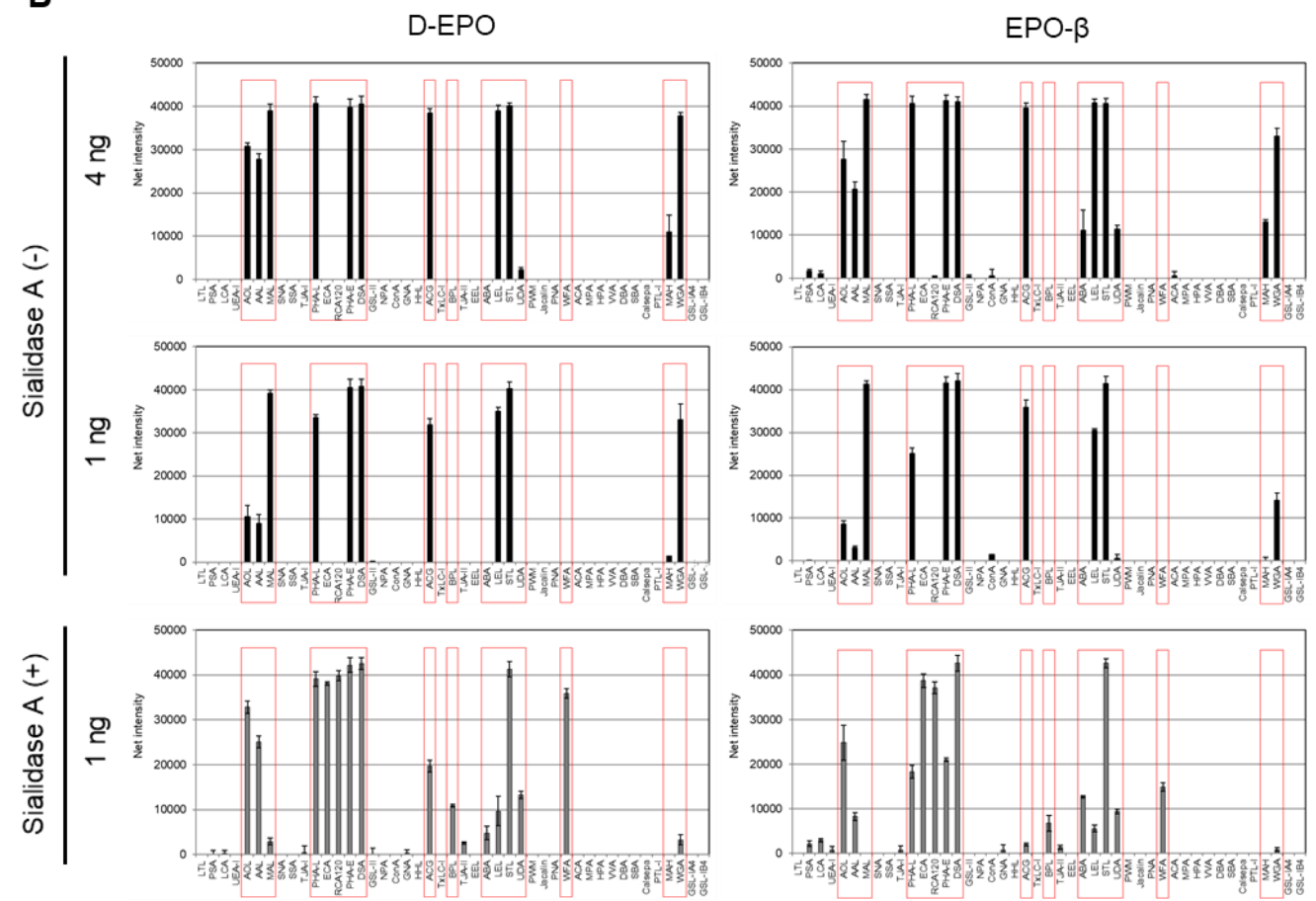

Figure S5. Sequential selection of lectins for GlycoBIST measurement of EPO. A: Scheme of selection steps. B: Lectin microarray data of two kinds of EPO with or without sialidase A treatment. The red oblongs indicate selected lectins. Data are represented as mean signals of three spots. Error bars indicate standard deviation. C: Results of a single lectin-antibody sandwich ELISA of EPO for four representative lectins. Data are shown as the average of duplicated wells. Error bars indicate standard deviation. 


\begin{tabular}{|c|c|c|c|c|c|c|c|c|}
\hline \multirow{3}{*}{$\begin{array}{l}\text { Lectin } \\
\text { name }\end{array}$} & \multirow{3}{*}{ Binding specificity ${ }^{\#}$} & \multicolumn{3}{|c|}{ Potential structures recognized by lectin ${ }^{* * *}$} & \multirow{2}{*}{\multicolumn{3}{|c|}{ Lectin microarray }} & \multirow{3}{*}{ GlycoBIST } \\
\hline & & \multicolumn{2}{|c|}{ N-glycan } & \multirow{2}{*}{ O-glycan } & & & & \\
\hline & & EPO- $\beta$ & D-EPO & & Intact & Sialidase A & PNGase $\mathrm{F}$ & \\
\hline $\mathrm{AOL}$ & $\begin{array}{l}\text { Fuca1-6GlcNAc (Core Fuc), Fuca } \\
\text { 1-2Galß1-4GIcNAc (H-type 2) }\end{array}$ & 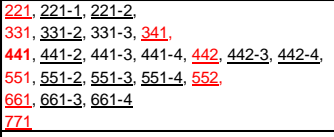 & $\begin{array}{l}221-2 \\
331, \underline{331-2}, 331-3, \\
441, \underline{441-2}, 441-3,441-4 \\
551, \underline{551-4}\end{array}$ & & $+/-$ & ++ & - & \\
\hline AAL & $\begin{array}{l}\text { Fuca1-3(Galß1-4)GlcNAc (Lewis } \\
\text { x), Fuca1-6GlcNAc (Core Fuc), }\end{array}$ & 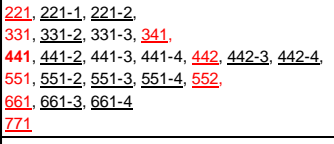 & $\begin{array}{l}221-2 \\
331, \underline{331-2}, 331-3, \\
441, \underline{441-2}, 441-3,441-4 \\
551, \underline{551-4}\end{array}$ & & + & ++ & - & \\
\hline MAL & Siaa2-3Galß1-4GIcNAc & 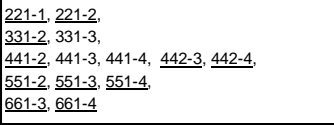 & $\begin{array}{l}\frac{221-2,}{331-2}, 331-3, \\
\frac{441-2}{451-4}, 441-3,441-4\end{array}$ & & ++ & - & - & $\bullet$ \\
\hline PHAL & $\begin{array}{l}\text { tri/tetra-antennary complex-type } \\
\mathrm{N} \text {-glycan }\end{array}$ & $\begin{array}{l}331, \underline{331-2}, 331-3, \underline{341}, \\
441, \underline{441-2}, 441-3,441-4, \underline{442}, \underline{442-3}, \underline{442-4}, \\
551, \underline{551-2}, \underline{551-3}, \underline{551-4}, \underline{552}, \\
\underline{661}, \underline{661-3}, \underline{661-4} \\
\underline{771}\end{array}$ & $\begin{array}{l}331, \underline{331-2}, 331-3, \\
441, \underline{441-2}, 441-3,441-4 \\
551, \underline{551-4}\end{array}$ & & ++ & ++ & - & $\bullet$ \\
\hline ECA & $\begin{array}{l}\text { Galß1-4GIcNAc (up with } \\
\text { increasing the number of terminal } \\
\text { Gal), no affinity for fully sialylated } \\
\text { N-type, fully agalactosylated N- } \\
\text { type }\end{array}$ & \begin{tabular}{|l}
$\frac{221,}{331,}$, \\
441,441, \\
551,552, \\
$\frac{661}{771}$, \\
71,
\end{tabular} & $\begin{array}{l}331, \\
441 \\
551\end{array}$ & & - & ++ & - & $\bullet$ \\
\hline RCA120 & $\begin{array}{l}\text { Galß1-4GIcNAc (up with } \\
\text { increasing the number of terminal } \\
\text { Gal), Galb1-3Gal (weak), no } \\
\text { affinity for agalactosylated N-type }\end{array}$ & $\begin{array}{l}\frac{221}{331}, \\
441,, 441, \\
551, \\
\frac{641}{572}, \\
771\end{array}$ & $\begin{array}{l}331 \\
441 \\
551\end{array}$ & & - & +++ & - & \\
\hline PHAE & $\begin{array}{l}\text { bi-antennary complex-type N- } \\
\text { glycan with outer Gal and } \\
\text { bisecting GlcNAc, no affinity for } \\
\text { fully sialylated N-type }\end{array}$ & 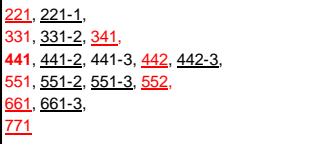 & $\begin{array}{l}\frac{221-2}{331, \underline{331-2}} \\
441, \underline{441-2}, 441-3 \\
551\end{array}$ & & ++ & ++ & - & - \\
\hline DSA & $\begin{array}{l}\text { (GlcNAcß1-4)n (Chitin), tri/tetra- } \\
\text { antennary N-glycan }\end{array}$ & 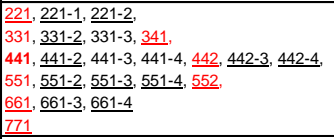 & $\begin{array}{l}221-2 \\
331, \underline{331-2}, 331-3, \\
441, \underline{441-2}, 441-3,441-4 \\
551, \underline{551-4}\end{array}$ & & ++ & ++ & - & - \\
\hline ACG & $\begin{array}{l}\text { Gal b1-3Gal, Siaa2-3Galß1- } \\
\text { 4GlcNAc }\end{array}$ & 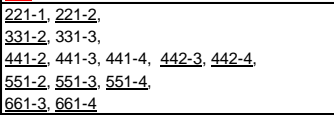 & $\begin{array}{l}\frac{221-2}{31-2}, 331-3, \\
\frac{341-2}{441-4}, 441-3,441-4 \\
\underline{551-4}\end{array}$ & $\mathrm{~T}$ & ++ & + & $+/-$ & \\
\hline BPL & $\begin{array}{l}\text { Galß1-3GalNAc (up with Lewis x, } \\
\text { down with Core Fuc), GalNAc }\end{array}$ & $\begin{array}{l}\frac{221}{331}, \underline{341}, \\
441, \underline{442}, \\
551, \underline{552} \\
\frac{661}{771} \\
\end{array}$ & $\begin{array}{l}331, \\
441 \\
551\end{array}$ & $\begin{array}{l}\text { Tn } \\
\text { T }\end{array}$ & - & $+/-$ & - & $\bullet$ \\
\hline ABA & Galß1-3GalNAc, GlcNAc & & & $\begin{array}{l}\text { T, ST, } \\
\text { (disialyI-T) }\end{array}$ & - & $+/-$ & - & - \\
\hline LEL & $\begin{array}{l}\text { (GIcNAcB1-4)n (Chitin), (Galb1- } \\
\text { 4GlcNAc)n (polylactosamine) }\end{array}$ & 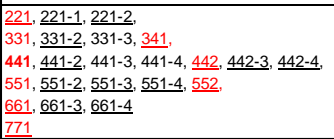 & $\begin{array}{l}221-2 \\
331, \\
441, \underline{331-2}, \underline{441-2}, 441-3, \\
551, \underline{551-4}\end{array}$ & & ++ & + & - & \\
\hline STL & $\begin{array}{l}\text { (GlcNAcB1-4)n (Chitin) } \\
\text { oligosaccharide containing } \\
\text { GlcNAc and MurNAc }\end{array}$ & 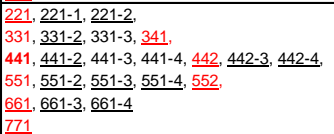 & $\begin{array}{l}221-2 \\
331, \underline{331-2}, 331-3, \\
441, \underline{441-2}, 441-3,441-4 \\
551, \underline{551-4}\end{array}$ & & ++ & + & - & \\
\hline UDA & $\begin{array}{l}\text { GlcNAcB1-4GIcNAc (Chitin), } \\
\text { High-Mannose (3 to High, up with } \\
\text { increasing the number of Man) }\end{array}$ & 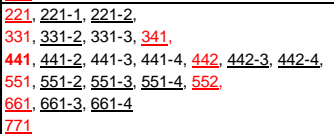 & $\begin{array}{l}221-2 \\
331, \underline{331-2}, 331-3, \\
441, \underline{441-2}, 441-3,441-4 \\
551, \underline{551-4}\end{array}$ & & ++ & ++ & - & \\
\hline WFA & $\begin{array}{l}\text { GalNAc } \beta 1-4 G I c N A c \text { (LacdiNAc), } \\
\text { Galß1-3(-6)GalNAc }\end{array}$ & $\begin{array}{l}\frac{221}{331}, \\
441, \underline{341} \\
551, \\
\frac{641}{552} \\
\underline{771} \\
\end{array}$ & $\begin{array}{l}331 \\
441 \\
551\end{array}$ & $\begin{array}{l}\mathrm{Tn} \\
\mathrm{T}\end{array}$ & - & + & - & $\bullet$ \\
\hline MAH & $\begin{array}{l}\text { Siaa2-3Galß1-3(Siaq2-6)GalNAc } \\
\text { (disialyI-T) }\end{array}$ & & & disialyl-T & - & - & - & - \\
\hline WGA & $\begin{array}{l}\text { (GlcNAcß1-4)n (Chitin), Hybrid } \\
\text { type N-glycan, Sia }\end{array}$ & 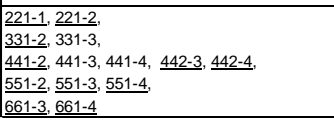 & $\begin{array}{l}\frac{221-2}{3}, \\
\frac{331-2}{441-2}, 331-3, \\
\frac{551-4}{4}\end{array}$ & & +++ & + & - & \\
\hline
\end{tabular}


Figure S6. Explanatory diagram for the relation of specificities of lectins binding to EPOs with EPO glycans estimated using MS (shown in Fig. S2). "Binding specificity of each lectin is shown as described previously ${ }^{1}$. $S$ tructures of $\mathrm{N}$ - and O-glycans were estimated by MALDI-TOF MS and Glyco-RIDGE, respectively. Glycan composition of $\mathrm{N}$-glycan is presented by digits (XYZ-S) indicating the numbers of Hex, HexNAc, $\mathrm{dHex}$ and NeuAc, respectively, on the trimannosyl core (Hex3HexNAc2=000). ${ }^{* *}$ Major $(>30 \%$ of the total intensity) and minor $(<10 \%)$ structures in EPO are bolded and underlined, respectively. Red indicates the glycan structure of EPO with sialidase A treatment. Further description on the change of lectin microarray signals with enzymatic treatments is inserted (+++: strong binding, ++: moderate binding, + : weak binding, +/-: trace binding, -: no binding). 


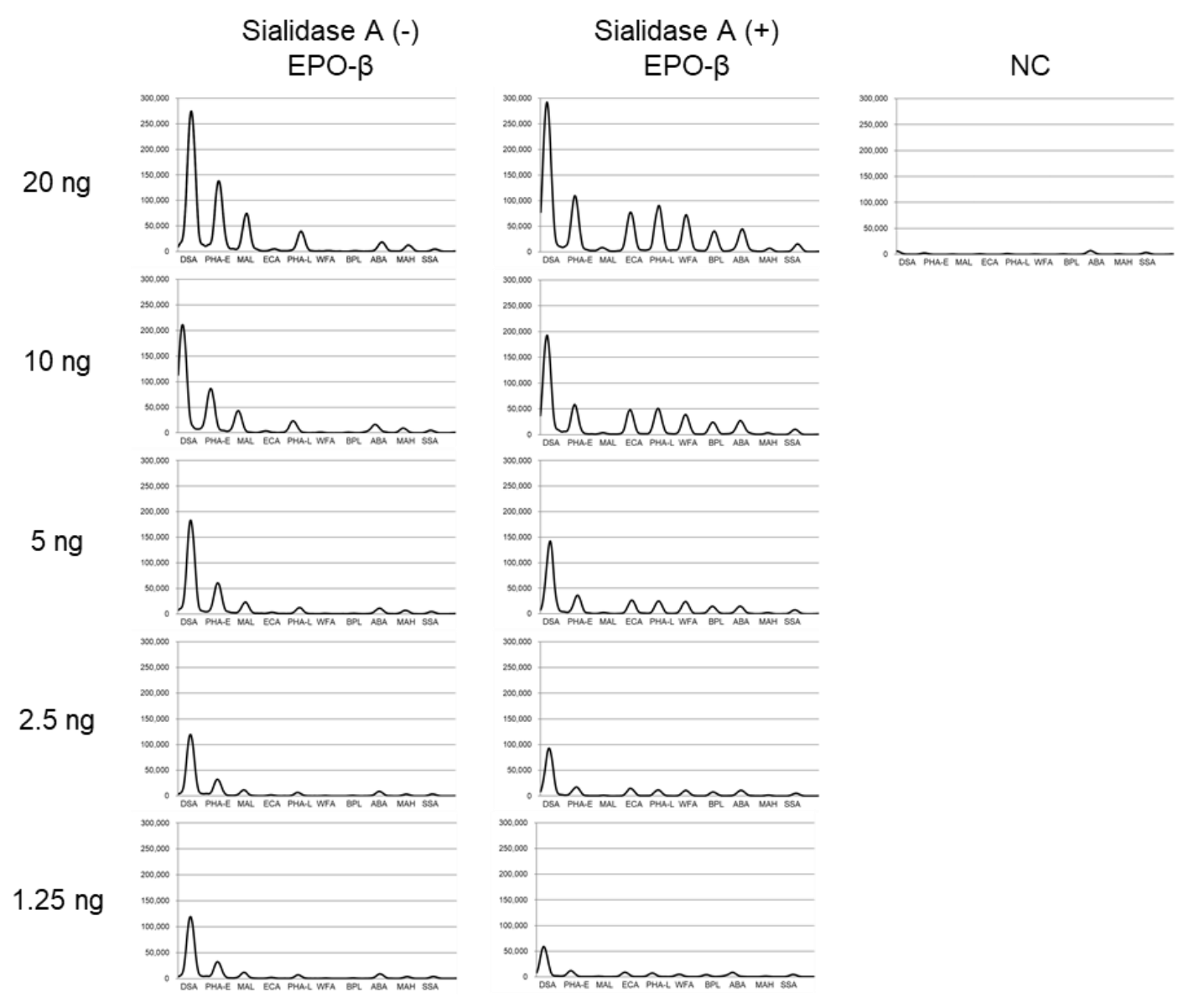

Figure S7. GlycoBIST scan data before processing (raw data for Fig. 3). The horizontal axis shows the position of the lectin in the tip and the vertical axis shows chemiluminescent signal intensity. Data are representative of one set (20-1.25 ng of EPO- $\beta$ with or without sialidase A treatment) among triplicate experiments. 
A
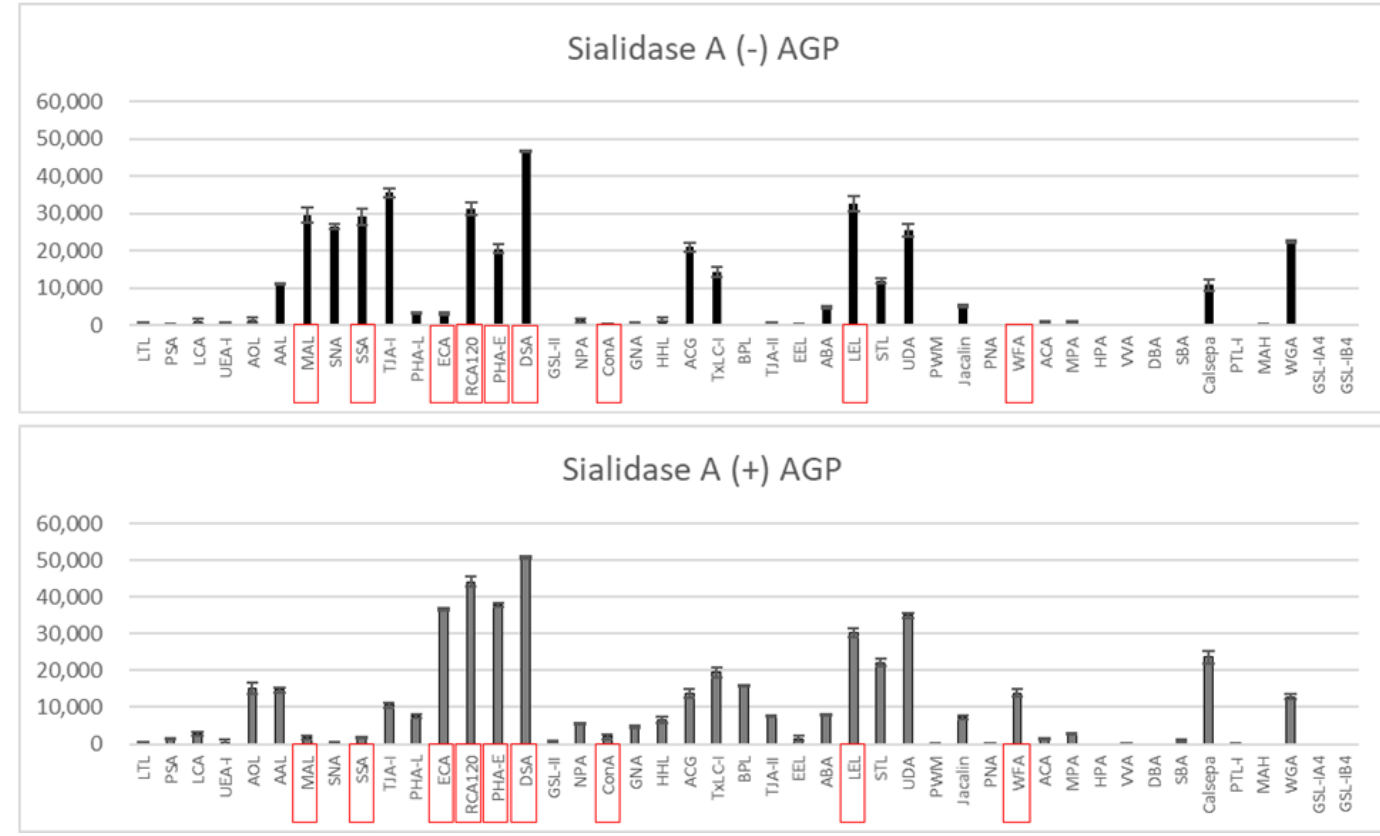

B
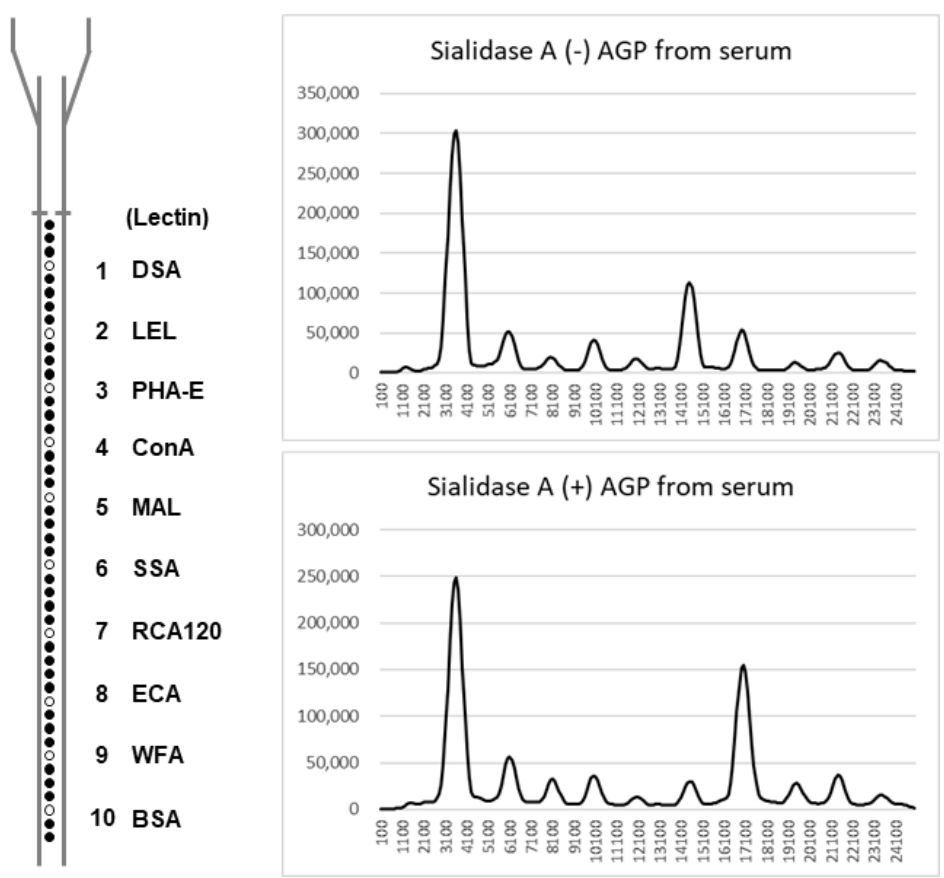

Figure S8. A: Lectin microarray data of endogenous AGP with or without sialidase A treatment. Red frames indicate selected lectins for measurement of GlycoBIST. B: GlycoBIST scan data before processing (raw data for Fig. 6A) and beads arrangement used for the measurement of AGP (left). Data are representative 1 pair among $n=8$ experiments. 
A

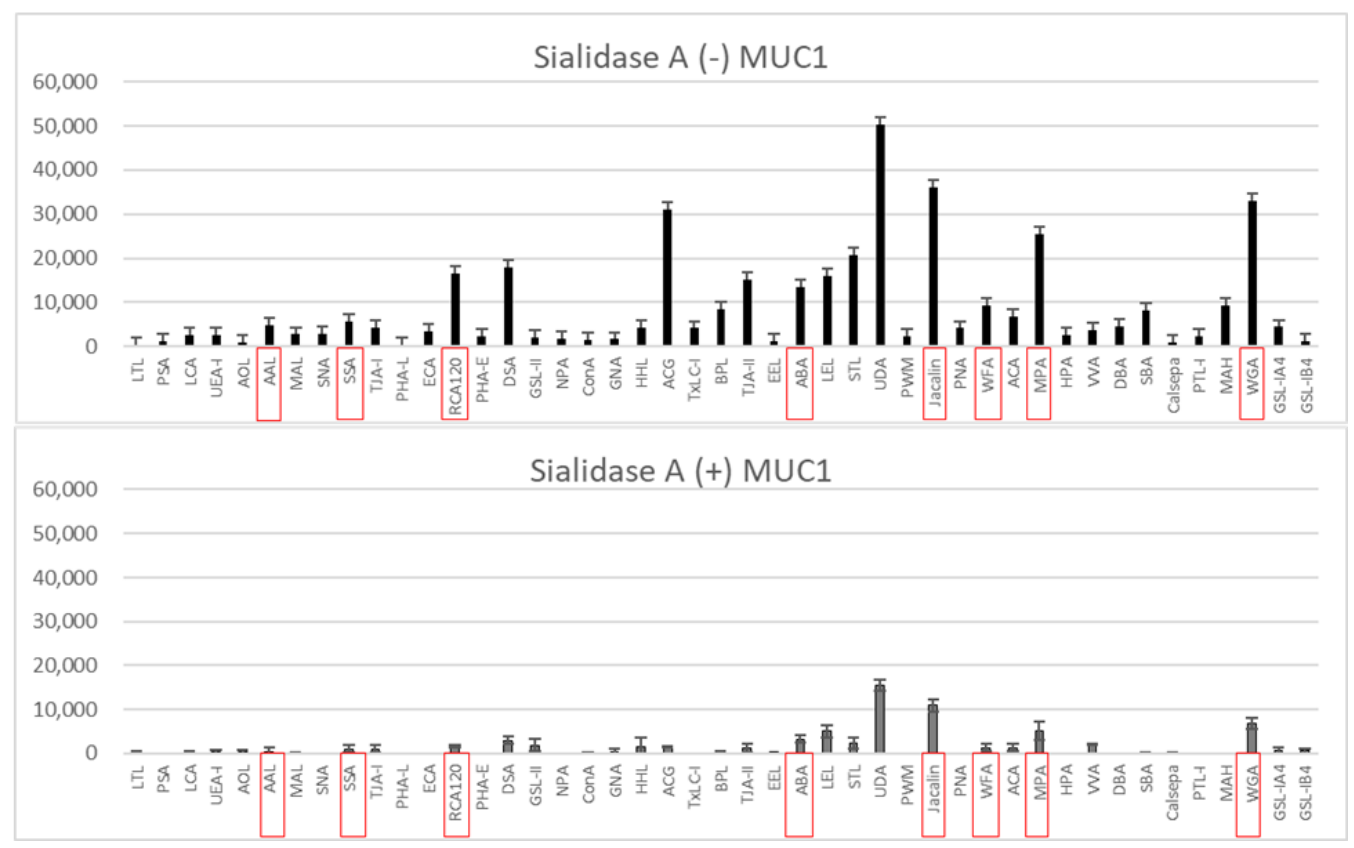

B

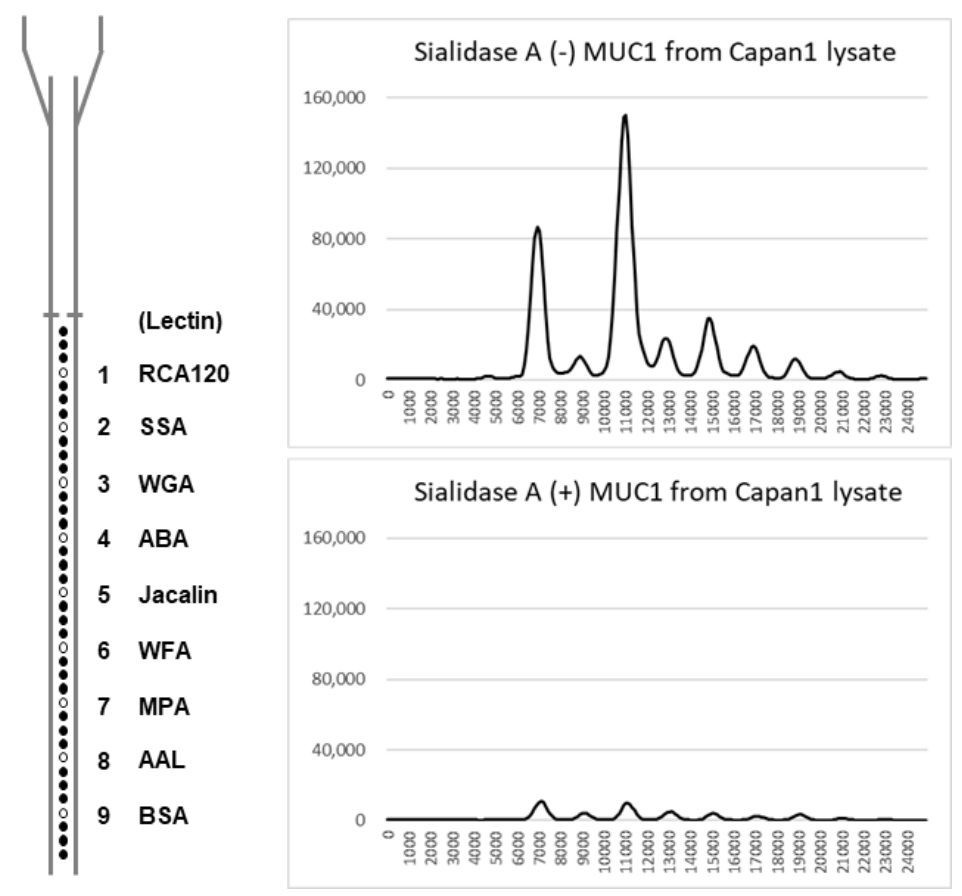

Figure S9. A: Lectin microarray data for membrane-tethered MUC1 with or without sialidase A treatment. Red frames indicate selected lectins for measurement of GlycoBIST. B: GlycoBIST scan data before processing (raw data for Fig. 6B) and bead arrangement used for the measurement of MUC1 (left). Data are representative 1 pair among $\mathrm{n}=8$ experiments. 


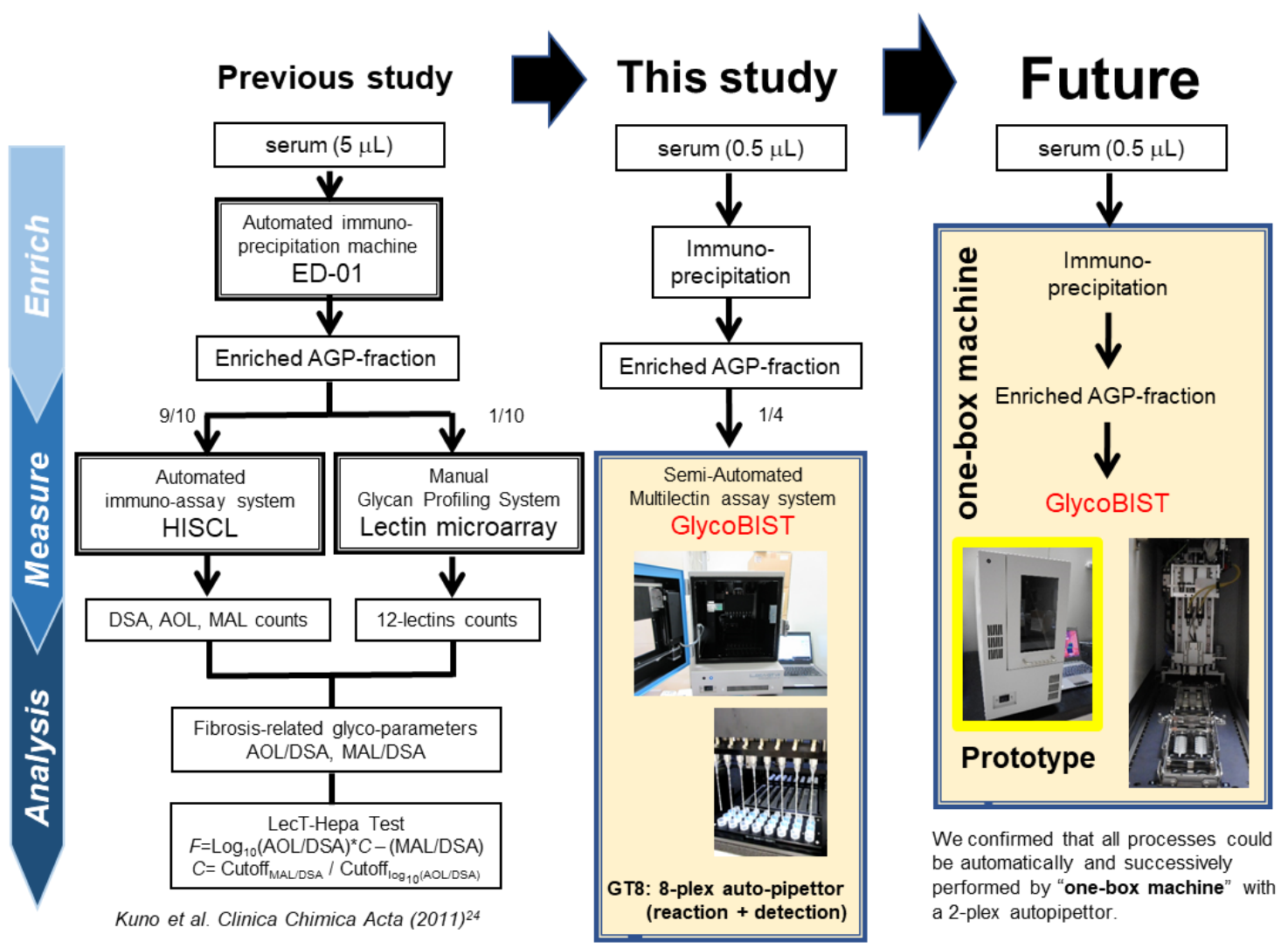

Figure S10. Process chart from sample treatment to glycan analysis for targeted glycoproteins. 


\section{References}

1) Nagai-Okatani C, Nagai M, Sato T, Kuno A. An Improved Method for Cell TypeSelective Glycomic Analysis of Tissue Sections Assisted by Fluorescence Laser Microdissection. Int J Mol Sci. 20(3), E700 (2019). 